\title{
Impact of Randomly Distributed Dopants on $\Omega$-Gate Junctionless Silicon Nanowire Transistors
}

\author{
Hamilton Carrillo-Nuñez ${ }^{\circledR}$, Muhamad M. Mirza, Douglas J. Paul, Senior Member, IEEE, \\ Donald A. MacLaren, Asen Asenov, Fellow, IEEE, and Vihar P. Georgiev, Member, IEEE
}

\begin{abstract}
This paper presents experimental and simulation analysis of an $\Omega$-shaped silicon junctionless nanowire field-effect transistor (JL-NWT) with gate lengths of $150 \mathrm{~nm}$ and diameter of the Si channel of $8 \mathrm{~nm}$. Our experimental measurements reveal that the oN-currents up to $1.15 \mathrm{~mA} / \mu \mathrm{m}$ for $1.0 \mathrm{~V}$ and $2.52 \mathrm{~mA} / \mu \mathrm{m}$ for the 1.8-V gate overdrive with an off-current set at $100 \mathrm{nA} / \mu \mathrm{m}$. Also, the experiment data reveal more than eight orders of magnitude on-current to off-current ratios and an excellent subthreshold slope of $66 \mathrm{mV} / \mathrm{dec}$ recorded at room temperature. The obtained experimental current-voltage characteristics are used as a reference point to calibrate the simulations models used in this paper. Our simulation data show good agreement with the experimental results. All simulations are based on driftdiffusion formalism with activated density gradient quantum corrections. Once the simulations methodology is established, the simulations are calibrated to the experimental data. After this, we have performed statistical numerical experiments of a set of 500 different JL-NWTs. Each device has a unique random distribution of the discrete dopants within the silicon body. From those statistical simulations, we extracted important figures of merit, such as off-current and on-current, subthreshold slope, and voltage threshold. The performed statistical analysis, on samples of those $500 \mathrm{JL}-N W T s$, shows that the mean $I_{D}-V_{G s}$ characteristic is in excellent agreement with the experimental measurements. Moreover, the mean $I_{D}-V_{G s}$ characteristic reproduces better the subthreshold slope data obtained from the experiment in comparison to the continuous model simulation. Finally, performance predictions for the JL transistor with shorter gate lengths and thinner oxide regions are carried out. Among the simulated JL transistors, the configuration with $25-\mathrm{nm}$ gate length and 2-nm oxide thickness shows the most promising characteristics offering scalable designs.
\end{abstract}

Index Terms-Drift diffusion (DD), electronic transport, junctionless (JL) nanowire field-effect transistors (FETs) (JL-NWTs), random dopants, scattering mechanisms, silicon nanowire, simulations, variability.

Manuscript received January 11, 2018; revised March 14, 2018; accepted March 19, 2018. Date of publication April 2, 2018; date of current version April 20, 2018. This work was supported by the U.K. EPSRC under Project EP/P009972/1 and Project EP/N003225/1. The review of this paper was arranged by Editor A. Schenk. (Corresponding author: Hamilton Carrillo-Nuñez.)

H. Carrillo-Nuñez, M. M. Mirza, D. J. Paul, A. Asenov, and V. P. Georgiev are with the School of Engineering, University of Glasgow, Glasgow G12 8LT, U.K. (e-mail: hamilton.carrillo-nunez@ glasgow.ac.uk; vihar.georgiev@glasgow.ac.uk).

D. A. MacLaren is with the SUPA School of Physics and Astronomy, University of Glasgow, Glasgow G12 8UU, U.K.

Color versions of one or more of the figures in this paper are available online at http://ieeexplore.ieee.org.

Digital Object Identifier 10.1109/TED.2018.2817919

\section{INTRODUCTION}

$\checkmark$ ILICON nanowires have a wide spectrum of promising applications such as current field-effect transistors (FETs) [1], ion-selective nanosensors [2], imaging sensor [3], photovoltaics [4], energy conversion and storage [5], and qubits [6]. However, fabricating a nanowire with characteristics required for a specific application can pose some major challenges. For example, as the transistor dimensions are reduced, the ON-current and OFF-current ratio $\left(I_{\mathrm{ON}} / I_{\mathrm{OFF}}\right)$ decreases. Keeping this ratio high is critical, if we want to achieve high-speed operations of the transistors. Also, keeping the $I_{\mathrm{OFF}}$ current very low is essential for low-power application systems such as tablets and mobile phones. Hence, the aim for the technology, nowadays, is to provide as low as possible $I_{\mathrm{OFF}}$ without comprising the high value of $I_{\mathrm{ON}}$. The low $I_{\mathrm{OFF}}$ is one of the major challenges of the planar MOSFETs. Source-to-drain quantum tunnelling [7]-[9] or variability [10]-[12] is much more pronounced and deteriorate the $I_{\mathrm{ON}} / I_{\mathrm{OFF}}$ ratio in current ultrascaled devices. To overcome this problem, a variety of new architectures, including ultrathin silicon-on-insulator (SOI) [13]-[15], double gate [13], [16], FinFETs [17], [18], trigate [19], $\Omega$-gate [20], junctionless (JL) [21], and gate all-around nanowire FETs [22], have therefore been developed to improve the electrostatic control of the conducting channel. In those architectures, the surface surrounded by the gate is increased in relation to the channel volume, improving the electrostatic control.

In conventional planar MOSFETs, the drain current $\left(I_{D}\right)$ can be improved accordingly to the semiconductor mobility and gate length $\left(\mu / L_{\mathrm{CH}}\right)$ ratio. Specifically, $I_{D} \propto\left(\mu / L_{\mathrm{CH}}\right)$ $\left(V_{\mathrm{GS}}-V_{\mathrm{TH}}\right)^{2}$, where $V_{\mathrm{GS}}$ and $V_{\mathrm{TH}}$ are the gate and threshold voltages, respectively. When reducing $L_{\mathrm{CH}}, I_{D}$ is expected to increase. However, $I_{D}$ is limited by the negative impact on the mobility of the large doping concentrations needed to preserve the electrostatic integrity in the channel. In addition, in ultrathin body MOSFETs, surface roughness (SR) scattering is increased due to the strong electric field necessary to form an electron channel close to the $\mathrm{Si}-$ oxide interface [21], thus deteriorating $I_{D}$.

Efforts from the device community have been, therefore, devoted on researching new high-mobility materials [23], [24] or novel devices architectures [25]-[27]. Flat-band devices such as JL nanowire FETs (JL-NWTs) have gained substantial attention in recent years [25]-[27]. Contrary to the "standard" p-type or n-type MOSFET devices that operate in an inversion 
mode, the JL-NWTs work in a partial depletion mode. As a result, the current is flowing mainly through the body of the transistor creating a 3-D conducting channel far away from the $\mathrm{Si}-$ oxide interface. This can explain why the SR scattering is not the limiting mechanism for these nanowire FETs [28].

Being a normally ON device, the JL transistor acts as a gated resistor that pinches off the carrier density by applying a gate voltage or by selecting a gate metal with an appropriate workfunction. It becomes a depleted, normally OFF, device. When switched ON, the JL transistor is under flat-band conditions. Due to the resistive behavior of the channel $I_{D}=I_{\mathrm{ON}}$, with $I_{\mathrm{ON}}=q \mu N_{D} A_{\perp} V_{D} / L_{\mathrm{CH}}$, where $q$ is the electronic charge, $N_{D}$ is the doping concentration, $A_{\perp}$ is the channel conducting cross section area, and $V_{\mathrm{DS}}$ is the source-drain applied voltage. From the last expression, one may think that by increasing the doping concentration $N_{D}, I_{\mathrm{ON}}$ would be increased. However, $N_{D}$ cannot be arbitrarily large, because the semiconductor will become a nearly metallic system, making the channel depletion for a particular cross section very difficult. Larger doping concentrations would also be reflected on a higher number of random distributed dopants, leading to strong variability of $I_{D}-V_{\mathrm{GS}}$ characteristics. Taking into account the position and the number of the random dopant in the $\mathrm{Si}$ body is important to investigate the optimal performance of specific JL-NWTs. Indeed, this paper focuses on random distributed dopants and their impact on the current-voltage characteristics of the fabricated $\Omega$-gate Si nanowire FETs reported in [28].

For that purpose, this paper is organized as follows. In Section II, the fabrication procedure of JL $\Omega$-gate transistors is summarized. The simulation methodology is explained in Section III. Statistical analysis performed on samples over $500 \mathrm{JL}$ transistors, including devices with shorter gate lengths and thinner oxide regions, is presented in Section IV. Finally, the conclusion is presented in Section V.

\section{FABRICATION}

The fabrication process of the JL-NWT considered here is explained in detail in our previous works [28]-[31]. For completeness of this paper, however, we summarize the most relevant steps during the fabrication procedure as follows.

Transistors have been fabricated from 55-nm SOI wafers from SOITEC with a 145-nm buried oxide. The Si channel was implanted with phosphorus at $15 \mathrm{keV}$ to allow majority of dopants to sit at the bottom part of the channel with a dose of $4 \times 10^{15} \mathrm{~cm}^{-2}$ before being annealled at $950{ }^{\circ} \mathrm{C}$ for $90 \mathrm{~s}$ to provide an implanted doping density of $8 \times 10^{19} \mathrm{~cm}^{-3}$. Temperature-dependent Hall bar measurements on large samples [28] were used to determine that the activated dopant density was $4 \times 10^{19} \mathrm{~cm}^{-3}$. This is well above the Mott criteria for Si:P, implying that the bulk material is strongly metallic in electronic behavior [32] which is confirmed by the temperature dependence of the electronic properties [28]. The top $\mathrm{Si}$ was then etched to reduce the thickness for the smallest dimension nanowires before a Vistec VB6 electron beam lithography tool was used to pattern the nanowire using hydrogen silsesquioxane (HSQ) resist. Initially, HSQ resist was used as an mask to etch 55-nm nanowires with 24, 16, and

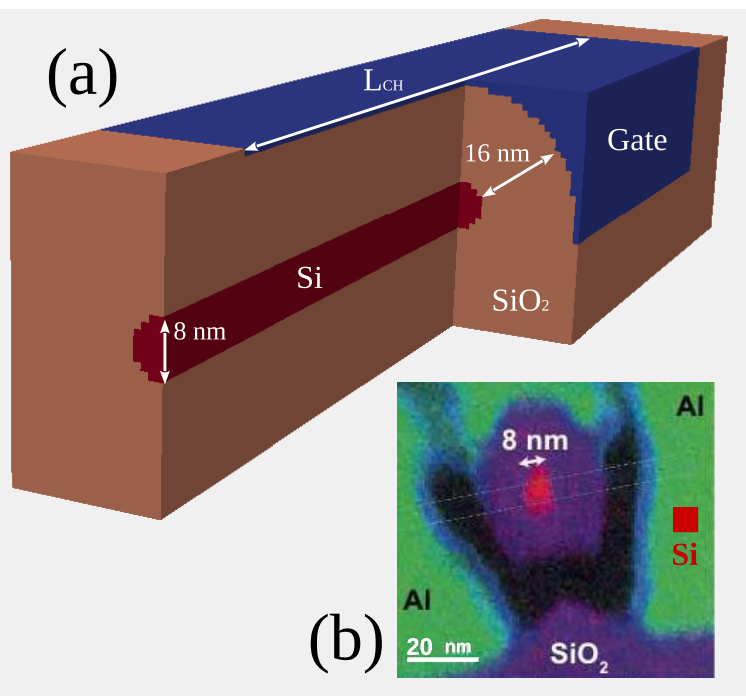

Fig. 1. (a) Sketch of the JL FET, with cuts along and perpendicular to the transport direction, considered in this paper. The gated channel region is designed following the $\Omega$-shaped of (b) fabricated silicon nanowire JLFET. The doping has been experimentally estimated to be $10^{19} \mathrm{~cm}^{-3}$. The silicon nanowire diameter is $8 \mathrm{~nm}$ and the oxide thickness is $16 \mathrm{~nm}$ within the $\Omega$-gated region with $150 \mathrm{~nm}$ long.

$8 \mathrm{~nm}$ widths, after which via holes were opened in polymethyl methacrylate (PMMA) resist to selectively thin down the $\mathrm{Si}$ channel. A low-damage $\mathrm{SF}_{6} / \mathrm{C}_{4} \mathrm{~F}_{8}$ inductivity coupled plasma etch [33] was undertaken before the resist was stripped and a thermal oxide grown at $950{ }^{\circ} \mathrm{C}$. Optical lithography was then used to define electrical contacts using $20 \mathrm{~nm}$ of $\mathrm{Ni}$ and $50 \mathrm{~nm}$ of $\mathrm{Pt}$ after the oxide had been stripped with HF. An anneal in forming gas at $380{ }^{\circ} \mathrm{C}$ for 15 min was used to alloy the contacts forming NiSi Ohmic contacts with a specific contact resistance of $0.8 \Omega \mathrm{mm}$. Finally, electron beam lithography was used with $400 \mathrm{~nm}$ of PMMA resist to lift-off the Al gate.

The oxidation step resulted in the nanowires being suspended above the buried oxide of the substrate preventing a short gate length being realized later in the fabrication process as reliable lift-off requires resist significantly thicker than any step height. A wide Al gate was, therefore, deposited by lift-off of total length of $2 \mu \mathrm{m}$ but since the nanowire length was $150 \mathrm{~nm}$, the effective gate length, $L_{g}$, is $150 \mathrm{~nm}$. The gate oxide of 16-nm equivalent oxide thickness for the devices has an integrated deep interface trap density, $D_{i t}$ below $10^{10} \mathrm{~cm}^{-2} \mathrm{eV}^{-1}$ as extracted from measurements on test capacitors fabricated on the same chips. An electron energy loss spectroscopy transmission electron microscope (TEM) image of the smallest nanowire with a diameter of $8 \pm 0.5 \mathrm{~nm}$ is presented in Fig. 1(b). A 3-D sketch of the fabricated and simulated JL-NWT is also shown in Fig. 1(a).

Direct current current-voltage characteristics were measured using an Agilent B1500 semiconductor parameter analyser at room temperature $(293 \mathrm{~K})$ with a Cascade Microtech probe station. For the alternating current (ac), lock-in measurements a constant voltage setup was used consisting of a $77-\mathrm{Hz} 0.1-\mathrm{V}$ amplitude ac sinusoidal signal from an Agilent 33521A function generator with a voltage 
divider (10 M $\Omega$ and $1 \mathrm{k} \Omega$ resistors) and the current measured using a 1-k $\Omega$ resistor with a Stanford Research SR830 lock-in amplifier.

\section{Simulation Methodology}

The JL transistors were simulated using the commercial TCAD simulator-GARAND which is now part of Synopsys TCAD Sentaurus Simulator. The solution of the transport problem is based on the drift-diffusion (DD) formalism. In this particular case, the DD approximation includes density gradient (DG) quantum confinement corrections [34]. The DG quantum correction is necessary to properly take into account quantum confinements effects within the DD approximation. The Coulomb potentials associated with individual discrete charges are also accurately captured by the DG, being important for the study in this paper. Finally, within this approximation one must solve self-consistently the Poisson and DG equations [35]. Fig. 1(a) represents the device considered in this paper. The silicon diameter is $8 \mathrm{~nm}$, the $\mathrm{SiO}_{2}$ body thickness is $16 \mathrm{~nm}$, and the gate length is $L_{\mathrm{CH}}=150$ $\mathrm{nm}$, which have been experimentally estimated and shown in Fig. 1(b). The cross section dimensions of the device are measured by a TEM, which clearly distinguishes the $\mathrm{Si}$ nanowire core (red) from its $\mathrm{SiO}_{2}$ (purple) surroundings. For the purpose of this paper, the channel cross section is fixed to $8 \mathrm{~nm}$, where the gate length and $\mathrm{SiO}_{2}$ are varied for performance predictions purpose. The source, channel, and drain regions are doped accordingly to the experimental value, being $N_{D}=10^{19} \mathrm{~cm}^{-3}$. The contacts, source, and drain are assumed to be semi-infinite. The von Neumann boundary conditions are imposed to allow the potential adjusts the electron injection to preserve charge neutrality in both of them. The device transport is then simulated at room temperature.

Fig. 2 compares the simulated current-voltage $\left(I_{D}-V_{\mathrm{GS}}\right)$ characteristics with its corresponding experimental measurements for six different devices all with $L_{\mathrm{CH}}=150 \mathrm{~nm}$ and oxide thickness of $16 \mathrm{~nm}$. In this case, the transistor was assumed to be uniformly doped which corresponds to continuous doping concentrations in the silicon. An agreement is achieved between the experimental values and the simulations results. The simulated $I_{D}-V_{\mathrm{GS}}$ curve is within the experimental variability at low- and high-gate voltage biases. The discrepancies in the subthreshold regime arise from the smooth and uniform nature of the doping profile where no imperfections, such as random dopants or oxide traps, have been considered. Also, in our simulations, an ideal $\Omega$-gate covers the Si channel. This is not the case for the fabricated devices, leading to poorer electrostatic integrity.

This agreement is obtained by choosing a combination of the low-field mobility (LFM), perpendicular fielddependent mobility (PFDM), and lateral field-dependent mobility (LFDM) models. Masetti et al. [36] dopingdependent model is used for the LFM. Yamaguchi [37] and Caughey and Thomas [38] models are then employed to compute the PFDM and LFDM. Calibration of the critical electric field $\left(F_{c}\right)$ and saturation velocity $\left(v_{\text {sat }}\right)$, respectively, for the last two models was carried out for the JL transistor

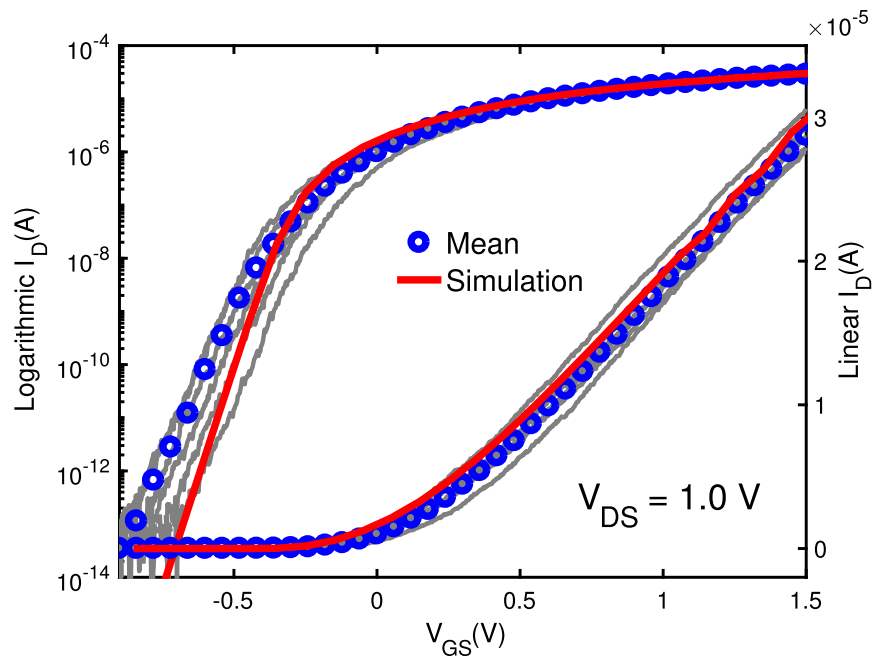

Fig. 2. Comparison of experimental and simulated (red line) $I_{D}-V_{G s}$ characteristics of the JL transistor shown in Fig. 1. Gray lines: experimental $I_{D}-V_{G S}$ characteristics from six different devices. Blue dotted curve: corresponding mean value. The gate length and oxide thickness are $L_{\mathrm{CH}}=150$ and $16 \mathrm{~nm}$, respectively. For calibration purpose, the $\mathrm{Si}$ region is uniformly doped with $N_{D}=10^{19} \mathrm{~cm}^{-3} \cdot I_{D}-V_{G s}$ characteristics are shown for (a) high $V_{D s}=1.0 \mathrm{~V}$ and (b) low $V_{D s}=5 \mathrm{mV}$. The calibration procedure is explained in Section III.

at high $V_{\mathrm{DS}}=1.0 \mathrm{~V}$. It was found that $F_{c}=5 \times 10^{7} \mathrm{~cm} / \mathrm{V}$, whereas $v_{\text {sat }}$ was set to $5 \times 10^{8} \mathrm{~cm} / \mathrm{s}$. The same values were used to simulate the JL transistor at low $V_{\mathrm{DS}}=5 \mathrm{mV}$, resulting in good agreement with the experimental measurement. It is worth mentioning that the values of these parameters are not universal. They might depend, for example, on the device dimensions as well as the doping concentration. They were kept fixed, however, for the simulations of JL transistors with random distributed dopants, presented in Section VI.

\section{Results}

Fig. 3 shows the impact of the random dopant distribution (RDD) on current-voltage $\left(I_{D}-V_{\mathrm{GS}}\right)$ characteristics at high drain bias. For the purpose of this analysis, 500 JL-NWTs with different RDDs were simulated. In all devices, the channel length was kept constant at $L_{\mathrm{CH}}=150 \mathrm{~nm}$ and the oxide thickness is also fixed at $16 \mathrm{~nm}$. The only difference for each device was the position and the number of the RDD in the source, the drain, and the channel. Based on the data presented in the Fig. 3, the following conclusions can be derived.

First, the ratio between the lowest and highest OFF-current $\left(I_{\mathrm{OFF}}\right)$ is $10^{9}$ or nine orders of magnitude. This variation in the values of the $I_{\mathrm{OFF}}$ current can be explained by establishing a correlation between the number and the position of the RDD in the Si region of their corresponding JL-NWTs. However, in case of the device with highest OFF-current, the dopants are equally spread all along the length of the Si body, as shown in Fig. 4(a). However, in the devices with the lowest $I_{\mathrm{OFF}}$ current, the dopants are more localized, forming clusters. In Fig. 4(b), one can observe dopants conglomerate in three well-defined groups in the source, in the beginning of the channel, and in the drain regions, leaving two isolated dopants at the channel-drain interface. In this case, the electrons coming 


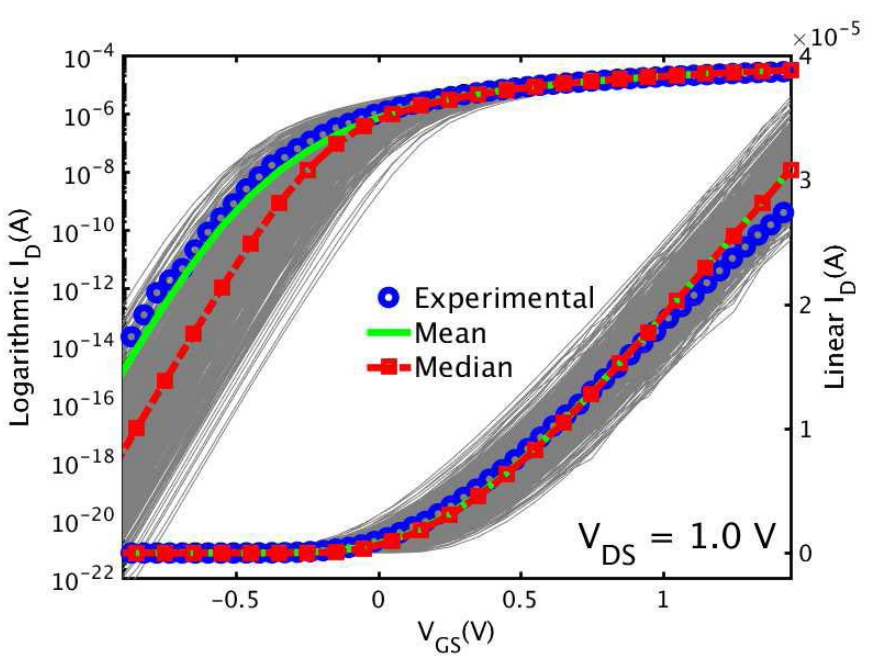

Fig. 3. $I_{D}-V_{G}$ characteristics of over $500 \mathrm{JL}$ transistors with random distributed dopants (gray curves). Dotted circle: experimental mean. Green line: statistical mean. Red dashed line: median. The last two are identical in the high gate bias region, e.g., $V_{G}>0 \mathrm{~V}$, and in good agreement with the experimental data. Within the subthreshold regime, the statistical mean current remains closer to the experimental measurements. The gate length is $150 \mathrm{~nm}$ whereas the oxide thickness is set to $16 \mathrm{~nm}$ in all devices. The doping concentration is as high as $N_{D}=10^{19} \mathrm{~cm}^{-3}$.

(a)
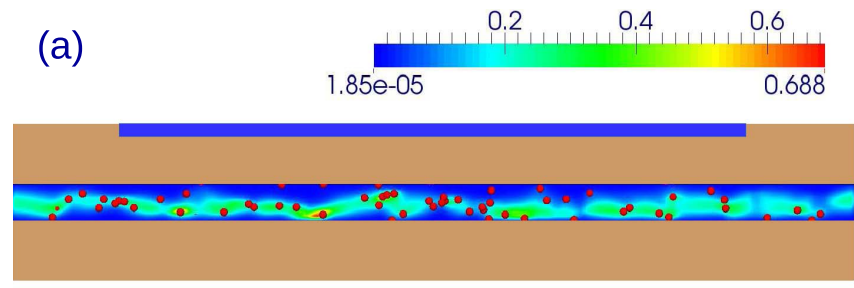

(b)



Fig. 4. Simulated 3-D dopant position and current density contours corresponding to JL transistors with (a) highest and (b) low OFF-currents. Current density units are $\mathrm{A} / \mu \mathrm{m}^{2}$. In case of the device with highest OFF-current, the dopants are more spread all along the Si channel, whereas for its counterpart in (b), dopants conglomerate in three well defined groups in the source, beginning of the channel, and drain regions, leaving two isolated dopants at the channel/drain interface. Such configuration generates less channels for electrons to go through the drain as observed in the current density contours, leading to lower OFF-state currents.

from the source need to overcome a higher potential barrier in this part of the transistor in comparison to the previous devices shown in Fig. 4(a), which is leading to a lower OFF-state current.

The corresponding current density contours shown in Fig. 4 reveal the percolation path which the electrons are kept moving from the source to the drain. Please note that the current flows is localized in the middle of the device which is exactly what is expected as the Fermi wavelength is $18.7 \mathrm{~nm}$ [31]. Also, even though the two currents flow look very similar, the difference in the current density between the both devices is almost 10 orders of magnitude. Another difference between the two
JL-NWTs is the total number of the RDD. The transistor with the highest OFF-current has 114 RDD in comparison with 73 RDD in the lowest OFF-current device. The actual number of dopants in each of the $500 \mathrm{JL}$ transistors is chosen randomly from a Poisson distribution, with the mean equal to the experimental doping concentration. The dopants are then placed randomly using a probability rejection technique implemented in the transport tool employed for this paper. The reader can find more details about the approach in [35] and [39]. Moreover, in the highest $I_{\mathrm{OFF}}$ nanowire, the dopants are distributed in such a way that they provide an almost continuous percolation path for the electrons from the source to the drain. This leads to enhancement of the $I_{\mathrm{OFF}}$.

Fig. 3 also compares the simulated median and mean and the experimental mean $I_{D}-V_{\mathrm{GS}}$ characteristics. Both simulated median and mean currents are technically identical in the high bias region of the $V_{\mathrm{GS}}$ range and in agreement with the experimental mean value of the current. When reaching the subthreshold region, the mean $I_{D}-V_{\mathrm{GS}}$ characteristic deviates from its median counterpart. The mean current, however, remains in good agreement with the experimental data over the entire $V_{\mathrm{GS}}$ range shown in Fig. 3. Moreover, compared to the $I_{D}-V_{\mathrm{GS}}$ curve for uniformly doped transistor (Fig. 2), the simulated mean $I_{D}-V_{\mathrm{GS}}$ characteristic reproduces better the experimental mean current within the subthreshold region. Hence, for this specific JL-NWTs, the "smooth" device, which is based on continuous doping, is further away from the experimental mean current in comparison to the mean device. For this reason, all comments and discussions of the results in this section are based only on the mean value of the $I_{D}-V_{\mathrm{GS}}$ characteristics. Moreover, the excellent match between the mean value device and the experimental data proves that statistical simulations are indeed necessary to accurately reproduce the experimental results in such ultrascaled devices.

Performing statistical simulations could help us to extract valuable information about the process and devices variability. For example, Fig. 5 shows probability distribution functions (PDFs) of some of the most important figures of merit (FoM) for those 500 JL-NWTs in Fig. 3. The PDF describes the probability of a particular event or value occurring. Hence, from the PDF distribution of the subthreshold swing (SS), we could conclude that most of the devices would have SS around their theoretical limit at room temperature, i.e., $60 \mathrm{mV} / \mathrm{dec}$. The PDF corresponding to the ON-current $\left(I_{\mathrm{ON}}\right)$ shows the devices will deliver $I_{\mathrm{ON}}$ close to the experimental value of $2.8 \times 10^{-5} \mathrm{~A}$ when the $\mathrm{JL}$ transistor is at $V_{\mathrm{DS}}=1 \mathrm{~V}$. The OFF-diagonal figures in Fig. 5 show the different correlations between FoMs. For example, subthreshold voltage $\left(V_{\mathrm{TH}}\right)$ and OFF-current $\left(I_{\mathrm{OFF}}\right)$ are strongly negatively correlated. Such correlation corresponds to decrease in the $I_{\mathrm{OFF}}$ value when the $V_{\mathrm{TH}}$ increases and vice versa. Note that the $I_{\mathrm{OFF}}$ axes are in log scale to highlight its difference between each device. Partial correlation between the $I_{\mathrm{ON}}$ and the other FoM can also be observed, except for the SS. Indeed, the correlation between SS and the rest of the FoM was found to be very low, as demonstrated in the last column of Fig. 5.

Next, we will study how the JL transistors would perform when reducing both the gate length and the oxide thickness. 


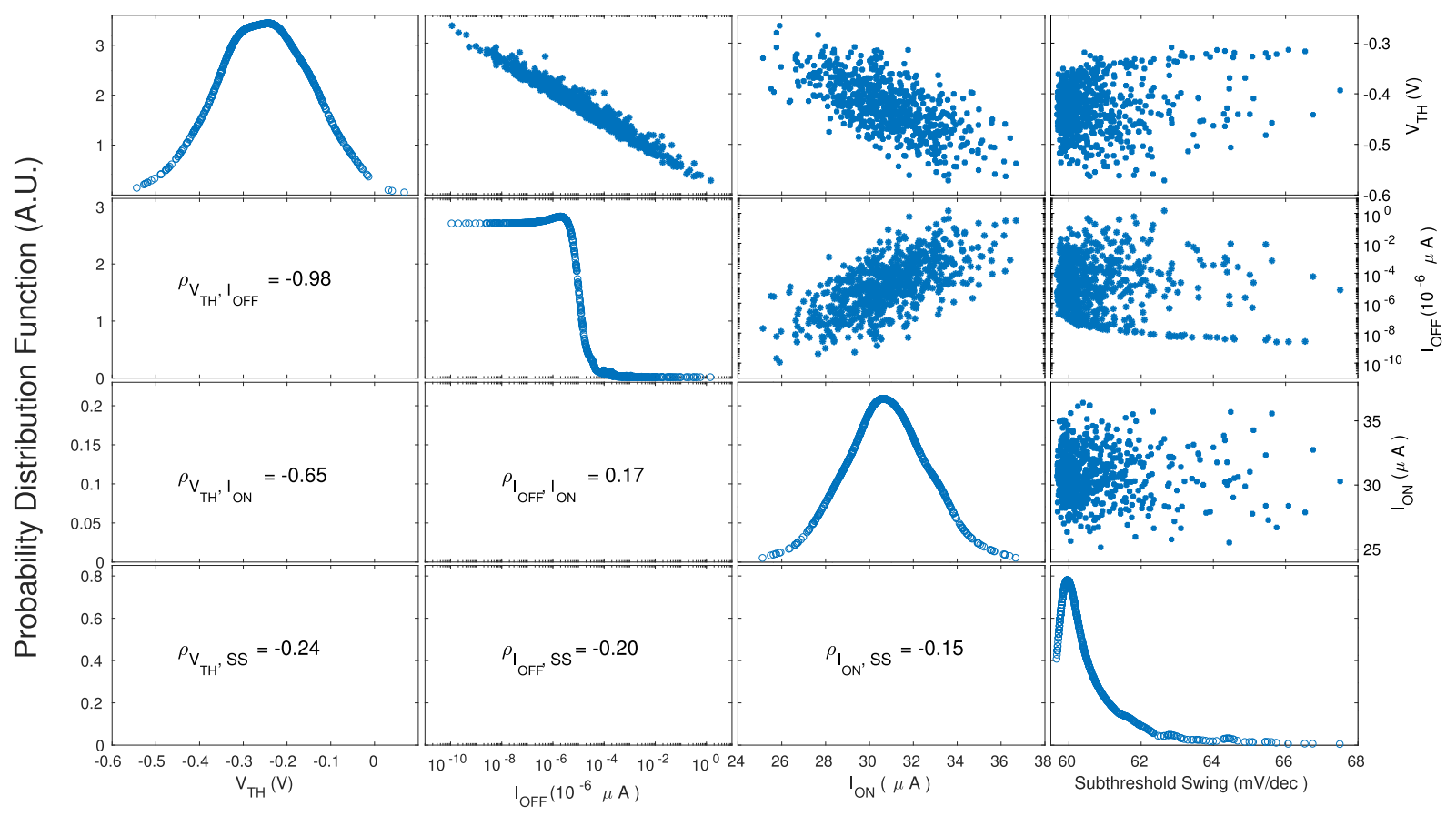

Fig. 5. PDFs and correlation between some of the most important FoM obtained from the simulation of those $500 \mathrm{JL}$ transistors shown in Fig. 3.

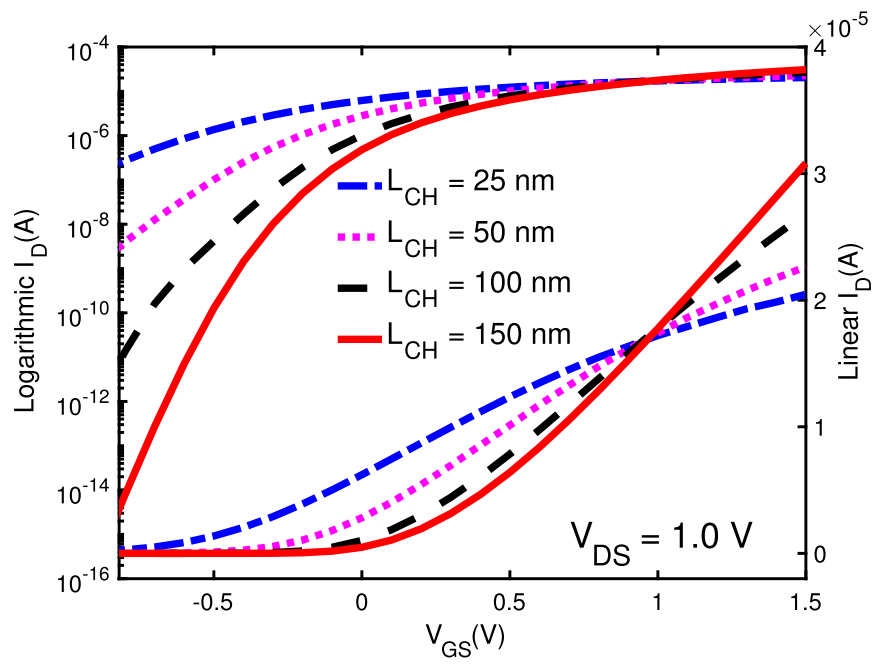

Fig. 6. Mean $I_{D}-V_{G s}$ characteristics of over $500 \mathrm{JL}$ transistor with different gate lengths varying from $L_{\mathrm{CH}}=150$ to $L_{\mathrm{CH}}=25 \mathrm{~nm}$. The oxide thickness is fixed to $16 \mathrm{~nm}$.

Over $500 \mathrm{JL}$ transistors with random distributed dopants were simulated for each gate length and oxide thickness. Due to the better agreement with experimental measurements presented for the JL transistor with $L_{\mathrm{CH}}=150 \mathrm{~nm}$ in Fig. 3, only mean $I_{D}-V_{\mathrm{GS}}$ characteristics for the simulated device will be reported from now on.

First, the oxide thickness is fixed to $16 \mathrm{~nm}$, while the gate length is shortened from $L_{\mathrm{CH}}=150$ to $L_{\mathrm{CH}}=25 \mathrm{~nm}$. The total length of the devices is kept fixed at $200 \mathrm{~nm}$. For example, in the case of the $L_{\mathrm{CH}}=25 \mathrm{~nm}$ device, the gate is exactly in the middle of the channel covering only $25 \mathrm{~nm}$ of the $200 \mathrm{~nm}$ long nanowire body. The same approach is used for all other gate lengths, where the gate is kept centered in the middle of the device. The corresponding mean $I_{D}-V_{\mathrm{GS}}$ characteristics for four different gate lengths are plotted in Fig. 6. From

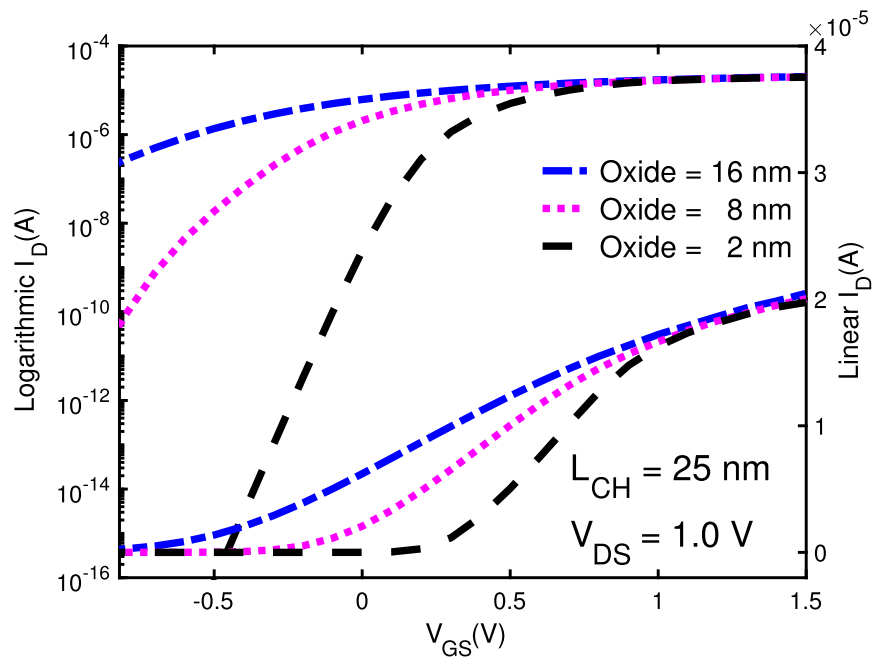

Fig. 7. Mean $I_{D}-V_{G s}$ characteristics of over $500 \mathrm{JL}$ transistor with different oxide thickness varying from 16 to $2 \mathrm{~nm}$. The gate length is fixed to $L_{\mathrm{CH}}=25 \mathrm{~nm}$.

the data, it is clear that the JL transistor performance is deteriorated when the gate length is reduced. The main reason is that by reducing the gate length, the electrostatic control is lost, leading to less steep $I_{D}-V_{\mathrm{GS}}$ characteristics with lower ON-current. In the worst case scenario, shown here, the ON-current is reduced by $30 \%$, while the SS increases up to approximately $200 \mathrm{mV} / \mathrm{dec}$ for transistors with $L_{\mathrm{CH}}=25 \mathrm{~nm}$. Second, the crossings of the $I_{D}-V_{\mathrm{GS}}$ curves at around $V_{\mathrm{GS}}=1.0 \mathrm{~V}$ occur due to the fact that when the gate length is decreased the wire behaves as a resistor. This can be compared to adding two resistors on both sides of the gate where the resistance increases with shortening the gate length.

Fig. 7 reports the mean $I_{D}-V_{\mathrm{GS}}$ characteristics of JL transistors with $L_{\mathrm{CH}}=25 \mathrm{~nm}$ and oxide thickness varying from 
16 to $2 \mathrm{~nm}$. A performance comparable with the fabricated JL transistor is predicted for the device with the thinnest oxide, showing the steepest slope with SS close to $60 \mathrm{mV} / \mathrm{dec}$ and the $I_{\mathrm{ON}} / I_{\mathrm{OFF}}$ ratio is greater than $10^{8}$. No improvement of the ON-current is observed. Hence, decreasing in the oxide thickness could indeed improve significantly the device behavior and allow the technology to be scaled down at least $L_{\mathrm{CH}}=25$ gate lengths. Finally, similar qualitatively results for correlations between the FoMs for JL-NWT with different gate lengths and oxide thickness were obtained.

\section{CONCLUSION}

In this paper, we report an investigation of JL-NWTs from the experimental and computational point of view. The experimental data revealed that JL devices with an 8-nm cross section and a 150-nm gate length demonstrate excellent transistorlike behavior with an SS of $66 \mathrm{mV} / \mathrm{dec}$ and a $10^{8}$ $I_{\mathrm{ON}} / I_{\mathrm{OFF}}$ ratio. All simulations in this paper were carried out using a DD solver, including DG quantum corrections. Agreement with experimental measurements was found after a careful calibration of mobility models.

A simulation study was performed to establish important device parameters and FoMs. This was achieved by simulating an ensemble of $500 \mathrm{JL}-\mathrm{NWTs}$ with different RDDs where $I_{D}-V_{\mathrm{GS}}$ characteristics for each device were obtained. Based on those $I_{D}-V_{\mathrm{GS}}$ characteristics, the mean and median values were extracted for each ensemble of devices with a different gate lengths. Our simulations showed that the JL-NWTs start to lose their electrostatic integrity when reducing the gate length below $50 \mathrm{~nm}$, degrading their performance, and that some important FoMs, such as the SS, are also effected. High-performance characteristics, however, could be recovered by significantly decreasing the oxide thickness. For example, if the oxide is scaled down to $2 \mathrm{~nm}$, the devices with the shortest gate length of 25-nm gate length still exhibits an excellent $I_{\mathrm{ON}} / I_{\mathrm{OFF}}$ ratio. We expect these results to allow optimizing nanowire transistors to improve their performance, which could be used for low-power applications and portable metrology.

\section{ACKNOWLEDGMENT}

The authors would like to thank the staff at the James Watt Nanofabrication Centre in Glasgow for their help in the fabrication of the devices.

\section{REFERENCES}

[1] Y. Guerfi and G. Larrieu, "Vertical silicon nanowire field effect transistors with nanoscale gate-all-around," Nanosc. Res. Lett., vol. 11, no. 1, p. 210, Dec. 2016. [Online]. Available: https://doi.org/10.1186/s11671016-1396-7

[2] P. Namdari, H. Daraee, and A. Eatemadi, "Recent advances in silicon nanowire biosensors: Synthesis methods, properties, and applications," Nanosc. Res. Lett., vol. 11, no. 1, p. 406, Dec. 2016. [Online]. Available: https://doi.org/10.1186/s11671-016-1618-z

[3] H. Park et al., "Filter-free image sensor pixels comprising silicon nanowires with selective color absorption," Nano Lett., vol. 14, no. 4, pp. 1804-1809, Apr. 2014. [Online]. Available: http://dx.doi.org/ $10.1021 / \mathrm{nl} 404379 \mathrm{w}$
[4] Y. Li, Q. Chen, D. He, and J. Li, "Radial junction Si micro/nanowire array photovoltaics: Recent progress from theoretical investigation to experimental realization," Nano Energy, vol. 7, pp. 10-24, Jul. 2014. [Online]. Available: http://www.sciencedirect.com/science/ article/pii/S2211285514000718

[5] K.-Q. Peng, X. Wang, L. Li, Y. Hu, and S.-T. Lee, "Silicon nanowires for advanced energy conversion and storage," Nano Today, vol. 8, no. 1, pp. 75-97, 2013. [Online]. Available: http://www.sciencedirect. com/science/article/pii/S1748013212001466

[6] F. A. Zwanenburg et al., "Silicon quantum electronics," Rev. Mod. Phys., vol. 85, pp. 961-1019, Jul. 2013. [Online]. Available: https:// link.aps.org/doi/10.1103/RevModPhys.85.961

[7] V. P. Georgiev, E. A. Towie, and A. Asenov, "Impact of precisely positioned dopants on the performance of an ultimate silicon nanowire transistor: A full three-dimensional NEGF simulation study," IEEE Trans. Electron Devices, vol. 60, no. 3, pp. 965-971, Mar. 2013. [Online]. Available: https://doi.org/10.1109/TED.2013.2238944

[8] M. Luisier, A. Schenk, and W. Fichtner, "Full-band atomistic study of source-to-drain tunneling in Si nanowire transistors," in Simulation of Semiconductor Processes and Devices. Vienna, Austria: Springer Vienna, 2007, pp. 221-224. [Online]. Available: https://doi.org/10.1007/ 978-3-211-72861-1 52

[9] H. Carrillo-Nuñez, M. Bescond, N. Cavassilas, E. Dib, and M. Lannoo, "Influence of electron-phonon interactions in single dopant nanowire transistors," J. Appl. Phys., vol. 116, no. 16, p. 164505, 2014. [Online]. Available: https://doi.org/10.1063/1.4898863

[10] A. Asenov et al., "Simulation of statistical variability in nano-CMOS transistors using drift-diffusion, Monte Carlo and non-equilibrium Green's function techniques," J. Comput. Electron., vol. 8, nos. 3-4, pp. 349-373, Oct. 2009. [Online]. Available: https://doi.org/10.1007/ s10825-009-0292-0

[11] T. Al-Ameri, V. P. Georgiev, F. Adamu-Lema, and A. Asenov, "Simulation study of vertically stacked lateral si nanowires transistors for 5-nm CMOS applications," IEEE J. Electron Devices Soc., vol. 5, no. 6, pp. 466-472, Nov. 2017. [Online]. Available: https://doi.org/10.1109/ JEDS.2017.2752465

[12] A. Asenov et al., "Nanowire transistor solutions for 5nm and beyond," in Proc. IEEE 17th Int. Symp. Quality Electron. Design (ISQED), Mar. 2016, pp. 269-274. [Online]. Available: https://doi.org/10.1109/ ISQED.2016.7479212

[13] H.-S. P. Wong, D. J. Frank, and P. M. Solomon, "Device design considerations for double-gate, ground-plane, and single-gated ultrathin SOI MOSFET's at the $25 \mathrm{~nm}$ channel length generation," in IEDM Tech. Dig., vol. 98. Dec. 1998, pp. 407-410. [Online]. Available: http://doi.org/10.1109/IEDM.1998.746385

[14] D. Esseni, M. Mastrapasqua, G. K. Celler, C. Fiegna, L. Selmi, and E. Sangiorgi, "An experimental study of mobility enhancement in ultrathin SOI transistors operated in double-gate mode," IEEE Trans. Electron Devices, vol. 50, no. 3, pp. 802-808, Mar. 2003. [Online]. Available: http://doi.org/10.1109/TED.2002.807444

[15] J.-P. Colinge, "The SOI MOSFET: From single gate to multigate," in FinFETs and Other Multi-Gate Transistors. Boston, MA, USA: Springer, 2008, pp. 1-48. [Online]. Available: http://doi.org/10.1007/ 978-0-387-71752-4_1

[16] F. Gámiz, A. Godoy, C. Sampedro, N. Rodriguez, and F. Ruiz, "Monte Carlo simulation of low-field mobility in strained double gate SOI transistors," J. Comput. Electron., vol. 7, no. 3, pp. 205-208, 2008. [Online]. Available: http://dx.doi.org/10.1007/s10825-007-0163-5

[17] D. Hisamoto et al., "FinFET-a self-aligned double-gate MOSFET scalable to $20 \mathrm{~nm}$," IEEE Trans. Electron Devices, vol. 47, no. 12, pp. 2320-2325, Dec. 2000. [Online]. Available: http://doi.org/10.1109/ 16.887014

[18] P.-L. Yang, T. B. Hook, P. J. Oldiges, and B. B. Doris, "Vertical slit FET at 7-nm node and beyond," IEEE Trans. Electron Devices, vol. 63, no. 8, pp. 3327-3334, Aug. 2016. [Online]. Available: http://doi.org/ 10.1109/TED.2016.2577629

[19] C.-H. Jan et al., "A $22 \mathrm{~nm}$ SoC platform technology featuring 3-D tri-gate and high-k/metal gate, optimized for ultra low power, high performance and high density SoC applications," in IEDM Tech. Dig., vol. 12. Dec. 2012, pp. 44-47. [Online]. Available: http://doi.org/ 10.1109/IEDM.2012.6478969

[20] F.-L. Yang et al., "25 nm CMOS omega FETs," in IEDM Tech. Dig., vol. 2. Dec. 2002, pp. 255-258. [Online]. Available: http://doi. org/10.1109/IEDM.2002.1175826

[21] J.-P. Colinge et al., "Nanowire transistors without junctions," Nature Nanotechnol., vol. 5, no. 3, pp. 225-229, 2010. [Online]. Available: http://dx.doi.org/10.1038/nnano.2010.15 
[22] N. Singh et al., "High-performance fully depleted silicon nanowire (diameter $\leq 5 \mathrm{~nm}$ ) gate-all-around CMOS devices," IEEE Electron Device Lett., vol. 27, no. 5, pp. 383-386, May 2006. [Online]. Available: http://dx.doi.org/10.1109/LED.2006.873381

[23] J. J. Gu, Y. Q. Liu, Y. Q. Wu, R. Colby, R. G. Gordon, and P. D. Ye, "First experimental demonstration of gate-all-around III-V MOSFETs by top-down approach," in IEDM Tech. Dig., vol. 11. Dec. 2011, pp. 769-773. [Online]. Available: http://doi.org/10.1109/IEDM.2011. 6131662

[24] S. W. Chang et al., "InAs N-MOSFETs with record performance of $\mathrm{I}_{o n}=600 \mu \mathrm{A} / \mu \mathrm{m}$ at $\mathrm{I}_{o f f}=100 \mathrm{nA} / \mu \mathrm{m}\left(\mathrm{V}_{d}=0.5 \mathrm{~V}\right), "$ in IEDM Tech. Dig., vol. 13. Dec. 2013, pp. 417-420. [Online]. Available: http://dx.doi.org/10.1109/IEDM.2013.6724639

[25] B.-H. Lee et al., "A vertically integrated junctionless nanowire transistor," Nano Lett., vol. 16, no. 3, pp. 1840-1847, Feb. 2016. [Online]. Available: http://dx.doi.org/10.1021/acs.nanolett.5b04926

[26] F. Vaurette, R. Leturcq, S. Lepilliet, B. Grandidier, and D. Stiévenard, "Confinement-modulated junctionless nanowire transistors for logic circuits," Nanoscale, vol. 6, no. 22, pp. 13446-13450, 2014. [Online]. Available: http://dx.doi.org/10.1039/C4NR04047C

[27] H. Wang, W. Han, L. Ma, X. Li, and F. Yang, "Investigation of mobility enhancement of junctionless nanowire transistor at low temperatures," J. Vac. Sci. Technol. B, Nanotechnol. Microelectron., Mater., Process., Meas., Phenom., vol. 33, no. 4, p. 040603, 2015. [Online]. Available: https://doi.org/10.1116/1.4926629

[28] M. M. Mirza et al., "Determining the electronic performance limitations in top-down-fabricated Si nanowires with mean widths down to $4 \mathrm{~nm}$,' Nano Lett., vol. 14, no. 11, pp. 6056-6060, 2014. [Online]. Available: http://dx.doi.org/10.1021/n15015298

[29] C. Busche et al., "Design and fabrication of memory devices based on nanoscale polyoxometalate clusters," Nature, vol. 515 , no. 7528, pp. $545-549,2014$. [Online]. Available: http://dx.doi.org/ 10.1038 /nature 13951

[30] V. P. Georgiev et al., "Experimental and simulation study of silicon nanowire transistors using heavily doped channels," IEEE Trans. Nanotechnol., vol. 16, no. 5, pp. 727-735, Sep. 2017. [Online]. Available: https://doi.org/10.1109/TNANO.2017.2665691

[31] M. M. Mirza, F. J. Schupp, J. A. Mol, D. A. MacLaren, G. A. D. Briggs, and D. J. Paul, "One dimensional transport in silicon nanowire junctionless field effect transistors," Sci. Rep., vol. 7, no. 1, 2017, Art. no. 3004 [Online]. Available: http://dx.doi.org/10.1038/s41598-017-03138-5
[32] P. P. Edwards and M. J. Sienko, "Universality aspects of the metalnonmetal transition in condensed media," Phys. Rev. B, Condens. Matter, vol. 17, no. 16, pp. 2575-2581, Mar. 1978. [Online]. Available: http://link.aps.org/doi/10.1103/PhysRevB.17.2575

[33] M. M. Mirza et al., "Nanofabrication of high aspect ratio ( 50:1) sub$10 \mathrm{~nm}$ silicon nanowires using inductively coupled plasma etching," J. Vac. Sci. Technol. B, Nanotechnol. Microelectron., Mater., Process., Meas., Phenom., vol. 30, no. 6, p. 06FF02, 2012. [Online]. Available: http://dx.doi.org/10.1116/1.4755835

[34] A. Asenov et al., "Simulation of statistical variability in nanoCMOS transistors using drift-diffusion, Monte Carlo and nonequilibrium Green's function techniques," J. Comput. Electron., vol. 8, nos. 3-4, pp. 349-373, 2009. [Online]. Available: http://dx.doi.org/ $10.1007 / \mathrm{s} 10825-009-0292-0$

[35] A. Asenov, "Random dopant induced threshold voltage lowering and fluctuations in sub-0.1 $\mu \mathrm{m}$ MOSFET's: A 3-D 'atomistic' simulation study," IEEE Trans. Electron Devices, vol. 45, no. 12, pp. 2505-2513, Dec. 1998. [Online]. Available: http://dx.doi.org/10.1109/16.735728

[36] G. Masetti, M. Severi, and S. Solmi, "Modeling of carrier mobility against carrier concentration in arsenic-, phosphorus-, and boron-doped silicon," IEEE Trans. Electron Devices, vol. ED-30, no. 7, pp. 764-769, Jul. 1983. [Online]. Available: https://doi.org/10.1109/T-ED.1983.21207

[37] K. Yamaguchi, "Field-dependent mobility model for two-dimensional numerical analysis of MOSFET's," IEEE Trans. Electron Devices, vol. ED-26, no. 7, pp. 1068-1074, Jul. 1979. [Online]. Available: https:// doi.org/10.1109/T-ED.1979.19547

[38] R. E. Thomas, "Carrier mobilities in silicon empirically related to doping and field," Proc. IEEE, vol. 55, no. 12, pp. 2192-2193, Dec. 1967. [Online]. Available: https://doi.org/10.1109/PROC.1967.6123

[39] A. Asenov, G. Slavcheva, A. R. Brown, J. H. Davies, and S. Saini, "Increase in the random dopant induced threshold fluctuations and lowering in sub-100 nm MOSFETs due to quantum effects: A 3-D densitygradient simulation study," IEEE Trans. Electron Devices, vol. 48, no. 4, pp. 722-729, Apr. 2001. [Online]. Available: http://dx.doi.org/ $10.1109 / 16.915703$

Authors' photographs and biographies not available at the time of publication. 